\title{
Stroke and Myocardial Infarction: A Bidirectional Mendelian Randomization Study
}

\author{
Wenxian Sun' \\ Luyang Zhang' \\ Weishi Liu' \\ Mengke Tian' \\ Xin Wang' \\ Jing Liang' \\ Yuying Wang' \\ Lan Ding' \\ Lulu Pei ${ }^{1}$ \\ Jie $\mathrm{Lu}^{2}$ \\ Yuming $X u^{\prime}$ \\ Bo Song' \\ 'Department of Neurology, The First \\ Affiliated Hospital of Zhengzhou \\ University, Zhengzhou, Henan Province, \\ People's Republic of China; ${ }^{2}$ Department \\ of Epidemiology and Health Statistics \\ College of Public Health, Zhengzhou \\ University, Zhengzhou, Henan Province, \\ People's Republic of China
}

Background: Stroke and myocardial infarction (MI) are associated with each other, as demonstrated in observational studies. However, it is unclear whether this relationship is causal, and the purpose of this study was to explore the bidirectional causality between stroke and MI.

Methods: Causality between stroke and MI was assessed using two-sample Mendelian randomization (MR). All genetic instruments related to stroke (40,585 cases; 406,111 controls) and MI (43,676 cases; 128,199 controls) were derived from large published genomewide association study. The MR analysis was calculated with inverse-variance weighting, MR-Egger, weighted mode, weighted median, and simple mode methods, and sensitivity analyses are used to detect the heterogeneity or pleiotropy.

Results: Genetically predicted large-artery stroke (LAS) was causally related to higher odds of MI (odds ratio $[\mathrm{OR}]=1.13,95 \%$ confidence interval $[\mathrm{CI}]: 1.06-1.20, p=1.0 \times 10^{-4}$ ), and the causal effect of LAS on MI was significantly weakened (OR $=1.09,95 \% \mathrm{CI}: 1.02-1.17, p=$ 0.017) after excluding the multipotent single-nucleotide polymorphisms (SNPs). MI phenotypes were genetically correlated with all ischemic strokes $(\mathrm{OR}=1.15,95 \% \mathrm{CI}: 1.03-1.28, p=0.013)$ and LAS $(\mathrm{OR}=1.39,95 \% \mathrm{CI}: 1.14-1.71, p=0.001)$; but a causal effect of MI on all ischemic strokes $(\mathrm{OR}=1.00,95 \% \mathrm{CI}: 0.95-1.28, p=0.219)$ and LAS $(\mathrm{OR}=1.26,95 \% \mathrm{CI}: 0.93-1.69, p=$ 0.130 ) was not observed after excluding the multipotent SNPs.

Conclusion: This MR analysis provides evidence to support the causal effect of LAS subtype on MI, and some factors act as confiding factors whereas others may act as mediators.

Keywords: causal inference, single-nucleotide polymorphism, genome-wide association study, epidemiologic methods, large-artery stroke

\section{Introduction}

Stroke and myocardial infarction (MI) are major clinical and public health problems. Both have a significant global impact; stroke leads to disability and mortality, ${ }^{1}$ and MI causes more than 7 million deaths worldwide annually. ${ }^{2}$

Observational studies confirmed that ischemic stroke and MI are interrelated. ${ }^{3}$ Patients with acute ischemic stroke have a high risk of MI, and the 5-year risk of MI after stroke is $8.6 \%$. ${ }^{4}$ Stroke causes autonomic dysfunction and systemic inflammation, and these dysregulation promote arrhythmia, microvascular dysfunction, coronary ischemia and myocardial necrosis. ${ }^{5,6}$ It has been reported that stroke is a serious complication after $\mathrm{MI},{ }^{7}$ with annual an incidence rate of up to $4 \% .{ }^{8}$ Left ventricle akinesia and/or dyskinesia after MI possibly increase the risk of mural thrombosis and cause stroke or peripheral thromboembolism. ${ }^{9}$ However, observational research has some potential biases, which may be caused by confounding factors or reverse causality. These biases cause the inability to form stable causal inferences in observational research when
Department of Neurology, The First Affiliated Hospital of Zhengzhou University, Ist Jianshe Eastern Road,

Zhengzhou, 450052, Henan Province,

People's Republic of China

Tel +86- | 3603983297

Fax +86-37I-66975I 40

Email fccsongb@zzu.edu.cn 
interpreting the results, ${ }^{10}$ so the causality behind these associations remains largely unclear. Thus, exploring new methods to prove the causal link between stroke and MI is important.

As a new epidemiological method, Mendelian randomization (MR) provides a stronger causal inference between exposure and disease risk. ${ }^{11} \mathrm{MR}$ analysis detects the causal relationship between exposure factors and outcomes by using genetic variation as instrumental variables (IVs). ${ }^{12} \mathrm{MR}$ can compensate for the shortcomings of traditional epidemiological studies that are affected by confounding factors and reverse causality. ${ }^{13}$ Recently, genome-wide association studies (GWASs) have been extensively performed and provides data resources for MR. ${ }^{14}$ The purpose of this study was to comprehensively explore the causality between ischemic stroke and MI using a two-sample bidirectional MR analysis.

\section{Materials and Methods}

\section{Study Design}

A two-sample MR study was performed to explore the potential causal relationship between ischemic stroke and its subtypes and MI. MR studies are based on three main assumptions (Figure 1A): ${ }^{15}$ IVs are related to exposure with genome-wide significance; the IVs are not related to any of the confounders associated with exposure or outcome; and the IVs influence outcome only through exposure. This study used a two-sample bidirectional MR method, and the design is shown in Figure 1B. In addition, we used the Strengthening the Reporting of Observational Studies in Epidemiology- Mendelian randomization $(\mathrm{STROBE}-\mathrm{MR})^{16}$ guidelines for reporting this study.

\section{Data Sources}

The diagnosis of ischemic stroke complied with the guidelines for early management of patients with acute ischemic stroke, ${ }^{17}$ and the diagnosis of MI in this study was based on the definition of American Heart Association (AHA) and American College of Cardiology (ACC). ${ }^{18}$ The data of ischemic stroke and its subtypes were acquired from GWASs of up to 446,696 individuals (40,585 cases; 406,111 controls) of European ancestry, conducted by the

A
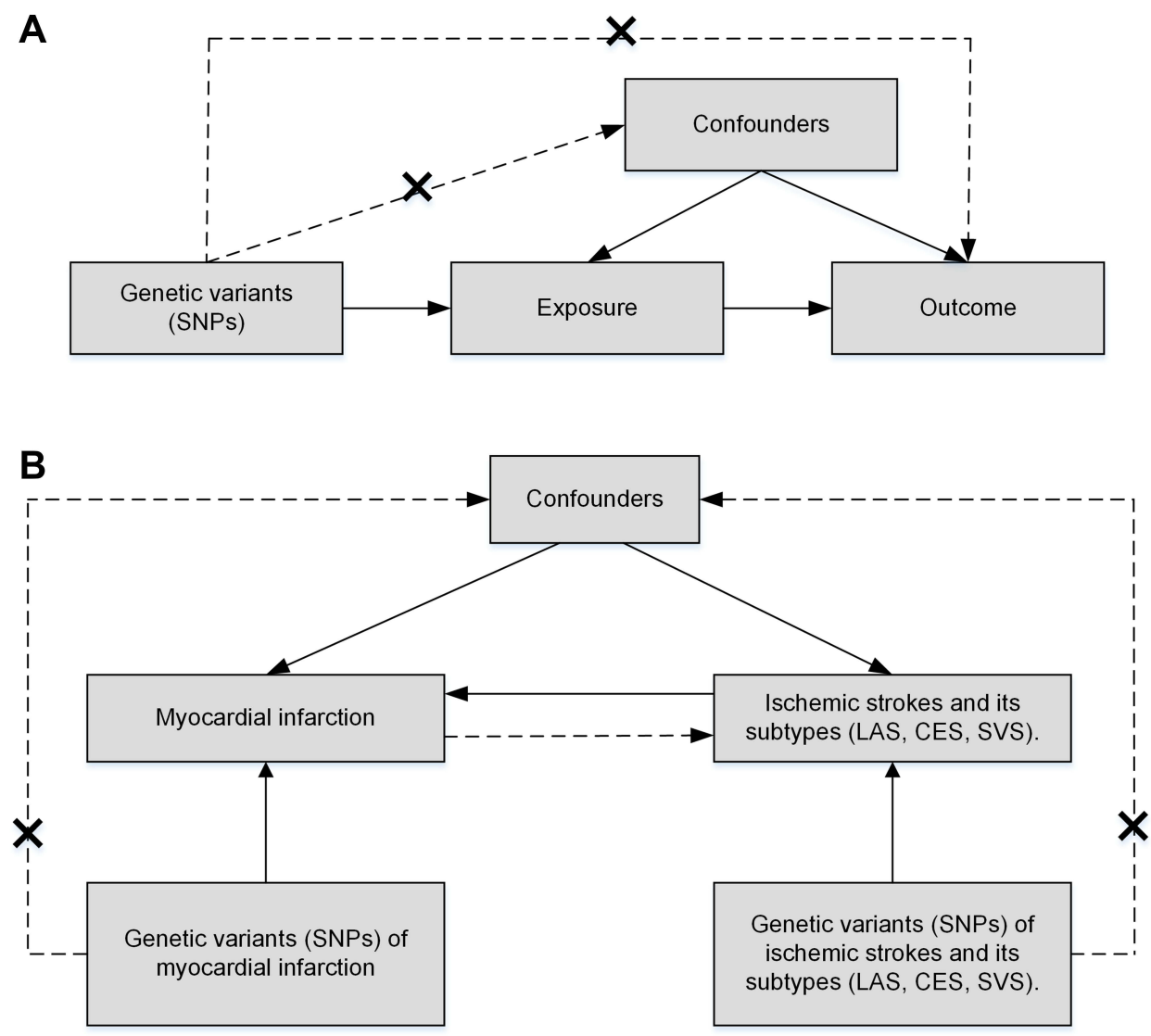

Figure I The main assumptions of two-sample Mendelian randomization and the design of this study. (A) The main assumptions of two-sample Mendelian randomization. (B) This study design of the Mendelian randomization analysis of ischemic stroke and myocardial infarction.

Abbreviations: LAS, large-artery stroke; CES, cardioembolic stroke; SVS, small vessel stroke; SNPs, single-nucleotide polymorphisms. 
MEGASTROKE consortium. ${ }^{19}$ Ischemic stroke was divided into three subtypes: large-artery stroke (LAS), cardioembolic stroke (CES), and small vessel stroke (SVS). Data on MI were acquired from a large GWAS meta-analysis with 171,875 subjects $(43,676$ cases; 128,199 controls) of European ancestry, conducted by the CARDIoGRAMplusC4D consortium. ${ }^{20}$ These original GWASs obtained ethical approval and informed consent from all participants.

\section{Single-Nucleotide Polymorphism (SNP) Selection}

All ischemic stroke and its subtypes were used as the exposure, and nine SNPs related to all ischemic stroke at genomewide significance $\left(p<5 \times 10^{-8}\right)$ were selected as genetic instruments. ${ }^{19}$ By testing linkage disequilibrium $\left(\mathrm{r}^{2}>0.01\right.$, $\mathrm{kb}<5000),{ }^{21}$ no SNP was excluded. To assess whether these SNPs were associated with confounding or risk factors for disease outcome, every SNP was searched in PhenoScanner $^{22,23}$ (http://www.phenoscanner.medschl.cam. ac.uk/) to exclude potential pleiotropy. Three SNPs (rs2634074, rs2107595, rs3184504) were associated with hypertension, type II diabetes, atrial fibrillation, and coronary heart disease and were excluded. Subsequently, we calculated the F-statistic of these SNPs (ranging from 74.0 to 150.6), indicating they were strong instruments for this MR study. ${ }^{24}$ The SNPs associated with ischemic stroke subtypes (LAS, CES, and SVS) were screened in the same way. Notably, there was no significant SNP $\left(p<5.0 \times 10^{-8}\right)$ in SVS; thus, we did not perform the analysis for SVS.

When MI was used as the exposure, 26 SNPs related to MI at genome-wide significance $\left(p<5 \times 10^{-8}\right)$ in the published GWAS $^{20}$ were used as genetic instruments. One SNP (rs10176176) was excluded by testing linkage disequilibrium. Subsequently, 25 SNPs were detected in PhenoScanner, and 13 SNPs (rs7528419, rs72689147, rs2327426, rs10947786, rs1510226, rs10455872, rs11556924, rs532436, rs653178, rs2681472, rs7173743, rs41290120, rs113113862) were associated with body mass index, current smoking, hypertension, type II diabetes, atrial fibrillation, low/high-density lipoprotein cholesterol or total cholesterol, and were excluded. The F-statistic of the selected IVs ranged from 11.0 to 124.4 , avoiding weak instrument bias.

\section{Statistical Analyses}

Statistical analyses of all data were performed using $\mathrm{R}$ (version 4.0.5) and a two-sample MR package. ${ }^{25}$ The main analysis was carried out by the random effects inverse-variance weighting (IVW) method, ${ }^{25}$ which provides consistent and efficient estimates when all genetic variants are valid. The weight of IVW comes from the reciprocal of the estimated variance of the Wald ratio of each SNP, and its weight represents the estimated accuracy of the Wald ratio of each SNP. The MR-Egger, weighted median, simple mode, and weighted mode regression methods were performed as complementary analyses. The MR-Egger method can provide robust estimates for situations that may violate standard IV assumptions due to directional pleiotropy. ${ }^{26}$ The weighted median method can provide robust estimates even if up to $50 \%$ of the information comes from ineffective IVs. ${ }^{27}$ In sensitivity analyses, we used the heterogeneity, pleiotropy, and leave-one-out tests. The heterogeneity test assessed the difference between each genetic variant. The MR-Egger intercept was used to assess horizontal pleiotropy, and horizontal pleiotropy, which was determined to exist if there was a large gap between the intercept term and zero. The leave-one-out analysis was conducted to test the robustness of the results by sequentially excluding one SNP at a time. The associations between genetically predicted exposure and outcome were presented as odds ratios (ORs) and 95\% confidence intervals (CIs). An observed two-sided $p$ values $<0.05$ were considered as significant evidence for a causal association, and a Bonferroni correction ( $p$-value after correction $=0.05$ / number of exposures/the number of outcomes) was used for multiple comparisons.

\section{Results}

We used two-sample bidirectional MR to analyze the causal relationship between $\mathrm{MI}$ and ischemic stroke. Genetically predicted stroke had a causal effect on MI but the causal effect of MI on stroke and its subtypes was not observed (Figure 1B). The detailed results were as follows.

\section{Effect of All Ischemic Stroke and Its Subtypes on the Risk of Myocardial Infarction}

All ischemic stroke and each of its subtypes were used as the exposure. There were nine available SNPs for all ischemic stroke, four SNPs for CES, four SNPs for LAS, and no available SNPs for SVS. The characteristics of these SNPs are shown in Online Supplemental Table 1. The main results 
of the MR analysis of the causal effect of stroke on MI are presented in Figure 2. The results showed a causal effect of all ischemic stroke on MI (odds ratio $[\mathrm{OR}]=1.31,95 \%$ confidence interval $[\mathrm{CI}]: 1.05-1.63, p=0.016)$, and LAS on $\mathrm{MI}\left(\mathrm{OR}=1.13,95 \% \mathrm{CI}: 1.06-1.20, p=1.0 \times 10^{-4}\right)$, but CES had no causal effect on MI (OR $=1.00,95 \%$ CI: 0.94 $1.06, p=0.942)$. The sensitivity analysis indicated heterogeneities among individual SNPs of all ischemic stroke on MI $\left(p=2.95 \times 10^{-5}\right)$, but there were no heterogeneities of LAS on MI $(p=0.355)$ and CES on MI $(p=0.939)$. The MREgger analysis showed that there was no horizontal pleiotropic effect: all ischemic stroke on MI (intercept $=-0.013$, $p=0.844)$, LAS on MI (intercept $=-0.017, p=0.424$ ), and CES on MI (intercept $=0.002, p=0.894$ ). By leave-one-out analysis, the results of all ischemic stroke (Figure $3 \mathrm{~A}$ ) and LAS (Figure 3B) on MI were robust and not significantly driven by any single SNP.

In addition, we analyzed the remaining six SNPs to observe the causal effect of all ischemic stroke on MI (Figure 2). The results demonstrated that the causal effect of all ischemic stroke on MI was significantly weakened using six SNPs $(\mathrm{OR}=1.15,95 \%$ CI: 1.00-1.33, $p=0.046$ ), which excluded SNPs associated with confounding factors. In the sensitivity analyses, this study did not find any heterogeneity $(p=0.598)$ or directional pleiotropy (intercept $=0.015, p=0.809$ ) for six SNPs. The effect of all ischemic stroke on MI was not substantially driven by any single SNP by leave-one-out analysis (Figure 3C). Regarding the effect of LAS on MI, the results (Figure 2) indicated the causal association between LAS and MI was significant with 3 SNPs (OR $=1.09$, 95\% CI: $1.02-1.17, p=0.017)$, and this causal relationship remained significant after Bonferroni correction (Bonferroni-corrected $p$-value: 0.017). The scatter plot in Figure 4 shows that the risk of MI increases as the genetically odds of LAS increase genetically. Moreover, sensitivity analyses confirmed there was no heterogeneity $(p=0.771)$ or pleiotropy (intercept $=$ $0.009, p=0.681$ ) for LAS on MI using three SNPs. The leave-one-out analysis (Figure 3D) demonstrated that the causal effect of LAS on MI is robust after eliminating confounding factors.

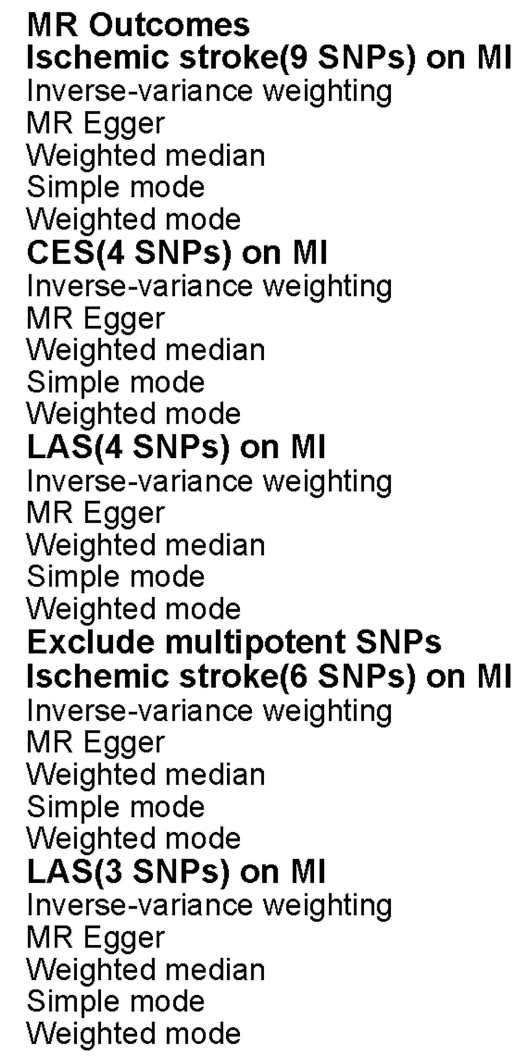

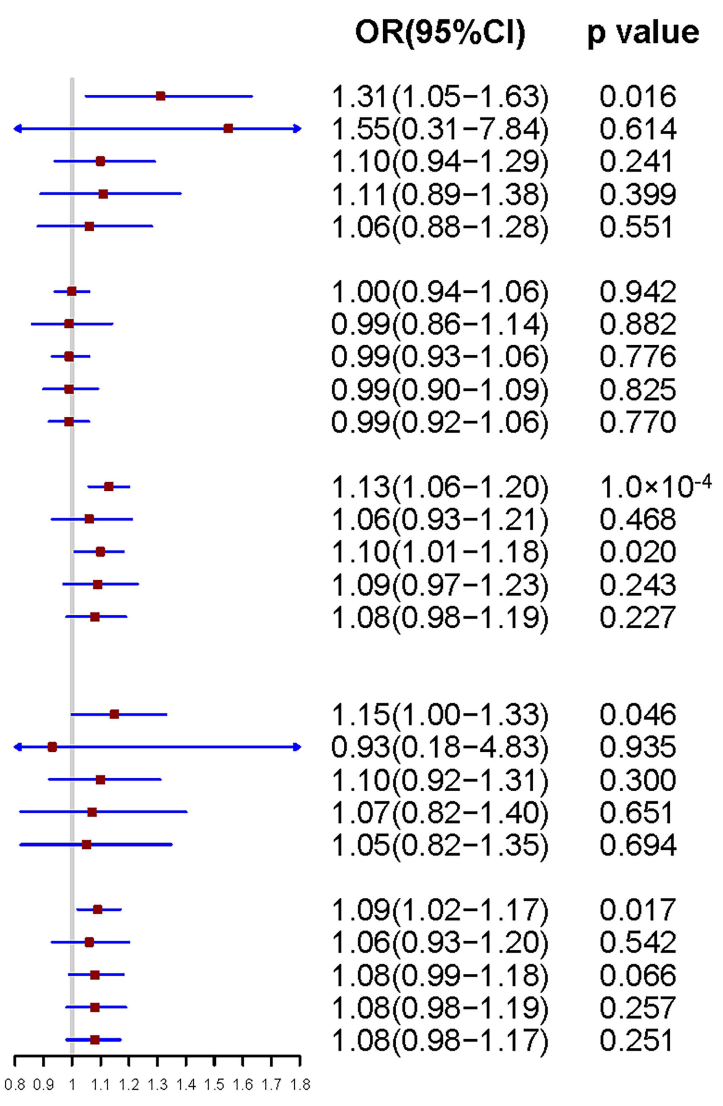

Figure 2 Mendelian randomization analysis of ischemic stroke and its subtypes for the risk of myocardial infarction. Abbreviations: OR, odds ratio; Cl, confidence interval; LAS, large-artery stroke; CES, cardioembolic stroke; IVW, inverse variance-weighted; MR, Mendelian randomization; MI, myocardial infarction; SNPs, single-nucleotide polymorphisms. 

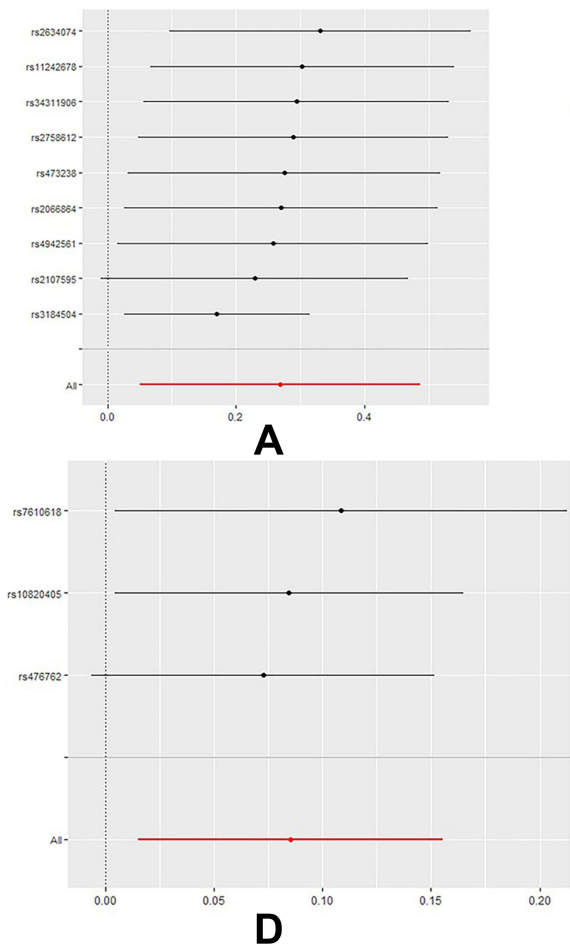

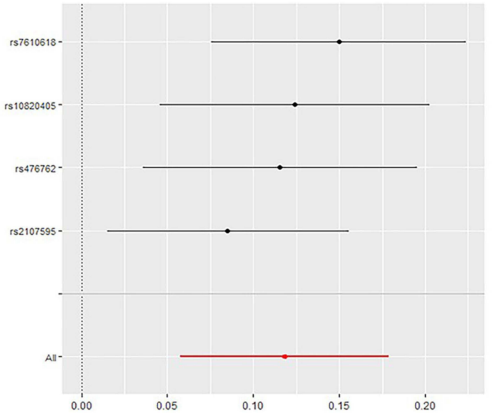

B

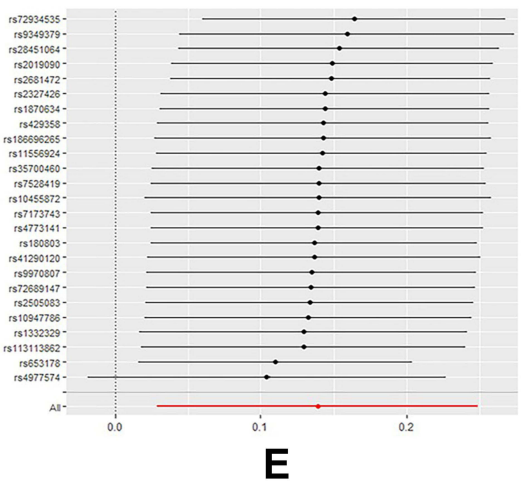

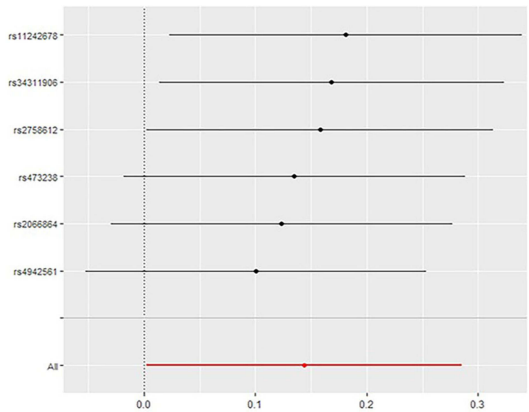

C

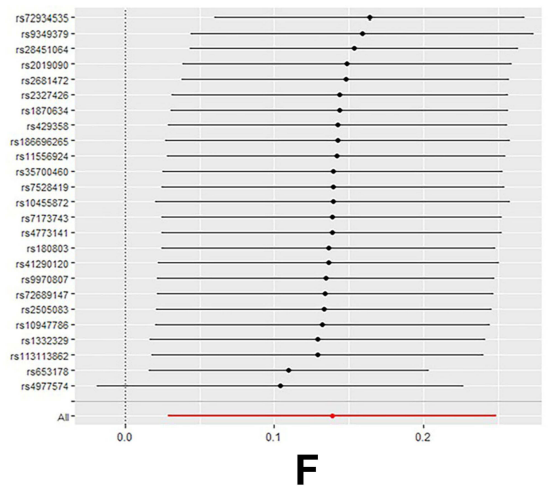

Figure 3 Mendelian randomization leave-one-out sensitivity analysis for exposure on outcome. (A) All ischemic stroke on myocardial infarction. (B) Large-artery stroke on myocardial infarction. (C) All ischemic stroke on myocardial infarction after excluding the multipotent SNPs. (D) Large-artery stroke on myocardial infarction after excluding the multipotent SNPs. (E) Myocardial infarction on all ischemic stroke. (F) Myocardial infarction on large-artery stroke. Circles indicate Mendelian randomization estimates for exposure on the outcome using the inverse-variance weighted method if the SNP was omitted. The bars indicate the confidence interval of Mendelian randomization estimates. Abbreviations: MR, Mendelian randomization; SNP, single-nucleotide polymorphism.
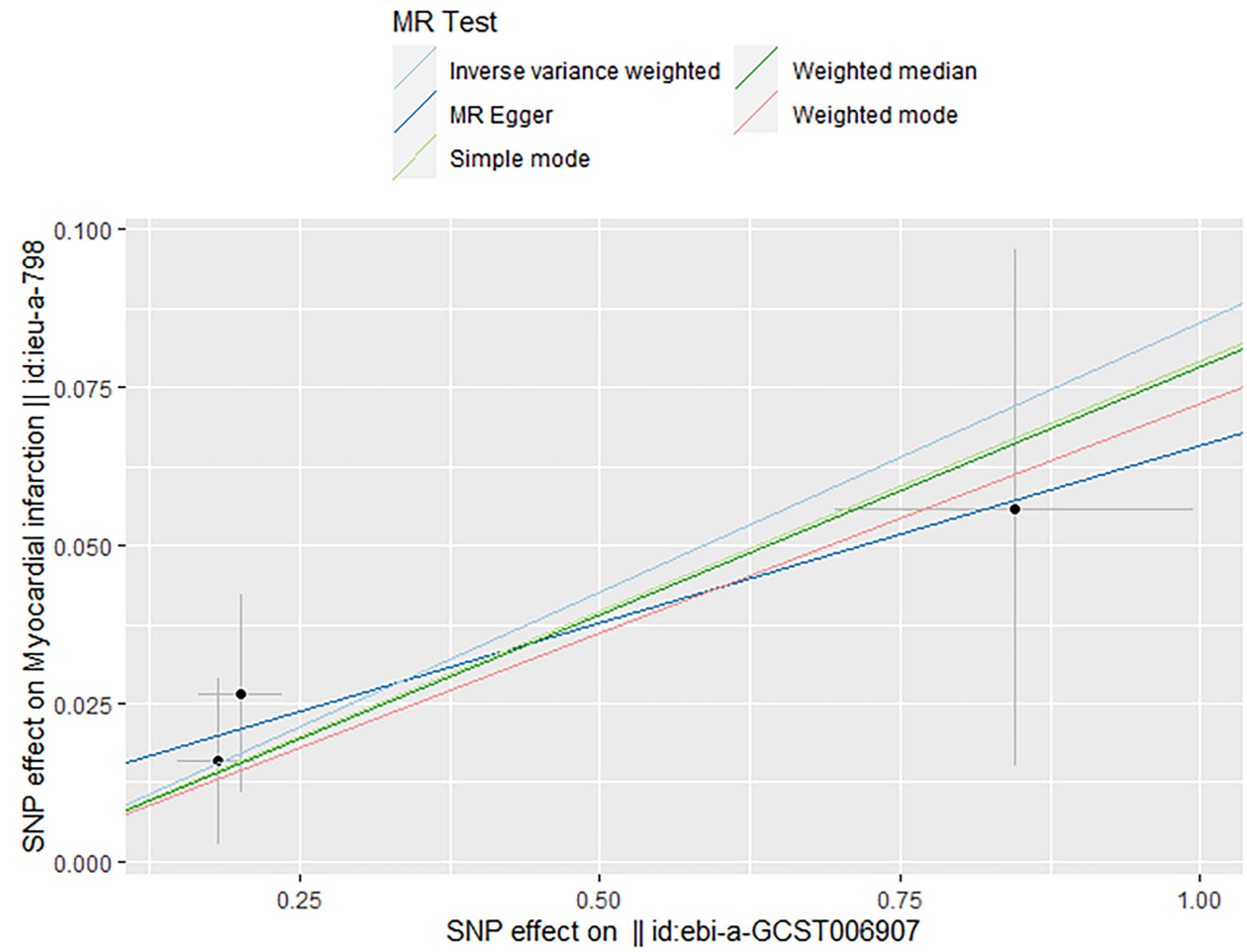

Figure 4 Associations of large-artery stroke related variants with risk of myocardial infarction. The line indicates the estimate of the effect using the MR method. Circles indicate marginal genetic associations with large-artery stroke and risk of myocardial infarction for each genetic variant. Error bars indicate $95 \%$ Cls. 
A

MR outcome
MI on stroke (25 SNPs)
Ischemic stroke
Inverse-variance weighting
MR Egger
Weighted median
Simple mode
Weighted mode
Cardioembolic stroke
Inverse-variance weighting
MR Egger
Weighted median
Simple mode
Weighted mode
Large artery stroke
Inverse-variance weighting
MR Egger
Weighted median
Simple mode
Weighted mode
Small vessel stroke
Inverse-variance weighting
MR Egger
Weighted median
Simple mode
Weighted mode

MR outcome

MI on stroke (25 SNPS)

Inverse-variance weighting

Egger

Simple mode

verse-variance weighting

Large artery stroke

Inverse-variance weighting

Simple mode

MR Egge

Simple mode

Weighted mode

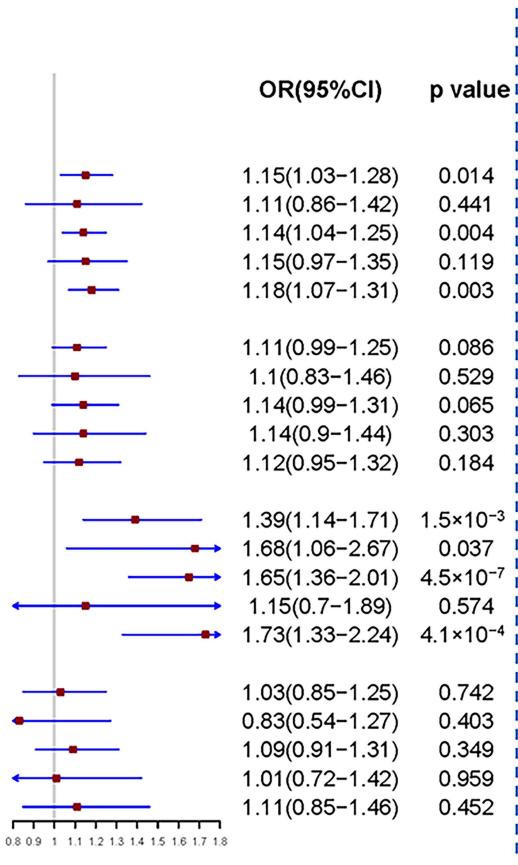

B

MR outcome

MI on stroke (12 SNPs)

Ischemic stroke

Inverse-variance weighting

MR Egger

Weighted median

Simple mode

Weighted mode

Cardioembolic stroke

Inverse-variance weighting

MR Egger

Weighted median

Simple mode

Weighted mode

Large artery stroke

Inverse-variance weighting

MR Egger

Weighted median

Simple mode

Weighted mode

Small vessel stroke

Inverse-variance weighting

MR Egger

Weighted median

Simple mode

Weighted mode

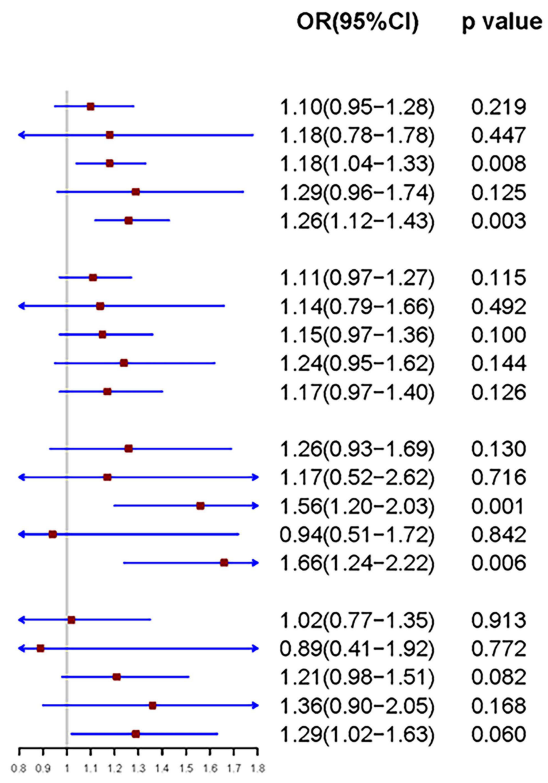

Figure 5 Mendelian randomization analysis of myocardial infarction for the risk of ischemic stroke and its subtypes. (A) The effect of all selected SNPs related to myocardial infarction on stroke and its subtypes. (B) The causal effect of myocardial infarction on stroke and its subtypes after excluding the multipotent SNPs.

Abbreviations: OR, odds ratio; $\mathrm{Cl}$, confidence interval; IVW, inverse variance-weighted; MR, Mendelian randomization; SNPs, single-nucleotide polymorphisms.

\section{Effect of Myocardial Infarction on Risk of All Ischemic Stroke and Its Subtypes}

When MI was used as the exposure, 25 eligible SNPs were obtained, and they are shown in Online Supplemental Table 2. The MR analysis of the causal effect of MI on ischemic stroke is presented in Figure 5A. The results revealed that the genetic prediction of MI was related to an increased risk of all ischemic stroke (OR $=1.15,95 \%$ CI: $1.03-1.28, p=0.014)$ and LAS $(\mathrm{OR}=1.39,95 \% \mathrm{CI}: 1.14-1.71, p=0.001)$. The causal effect of MI on CES (OR $=1.11,95 \%$ CI: $0.99-1.25, p=0.086)$ and SVS (OR $=1.03,95 \%$ CI: $0.85-1.25, p=0.742)$ was not significant. In the sensitivity analysis, the results indicated strong heterogeneities for all ischemic stroke $\left(p=5.21 \times 10^{-16}\right)$, LAS $\left(p=1.17 \times 10^{-6}\right)$, and SVS $\left(p=1.92 \times 10^{-6}\right)$, but not for CES $(p=0.032)$. There was no directional pleiotropy for the relationship between the SNPs and risk of all ischemic stroke (intercept $=0.004, p=0.748$ ), LAS (intercept $=-0.022$, $p=0.379$ ), CES (intercept $=0.001, p=0.924$ ), and SVS (intercept $=0.025, p=0.277$ ) in MR-Egger regression. The causal effect of MI on all ischemic stroke (Figure 3E) and LAS (Figure 3F) was not driven by any single SNP as indicated by leave-one-out analysis. After excluding the multipotent SNPs, the analysis results of the remaining 12 SNPS showed that MI had no causal effect on all ischemic stroke $(\mathrm{OR}=1.10,95 \% \mathrm{CI}$ : $0.95-1.28, p=0.219)$, on LAS (OR $=1.26,95 \%$ CI: $0.93-1.69$, $p=0.130)$, on CES (OR $=1.11,95 \%$ CI: $0.97-1.27, p=0.115)$ and on SVS (OR $=1.02,95 \%$ CI: $0.77-1.35, p=0.913)$ (Figure 5B).

\section{Discussion}

In this study, the primary two-sample MR analysis provided evidence that genetically increasing odds of LAS subtype has a causal relationship with increased risk of MI, whereas the causal effect of MI on stroke and its subtypes was not detected. Furthermore, we used different statistical models and instruments to demonstrate that these results are robust.

Patients with transient ischemic attack or stroke have a high risk of MI according to a meta-analysis, ${ }^{28}$ which included 39 studies with 65,996 patients. Through a multicenter stroke registry in Korea, two studies ${ }^{29,30}$ revealed that the 1-year and 5-year cumulative incidence rates of MI after acute ischemic stroke were $0.5 \%$ and $2 \%$, respectively. The Northern Manhattan Study showed that the 5-year risk of MI was $8.6 \%$ in patients with stroke, though more research is needed to detect the determinants of MI after stroke. ${ }^{4}$ However, due to the essential characteristics of observational studies, these studies still cannot control the effects of unmeasured confounding factors and reverse causality. This study observed that the causal 
effect of all ischemic stroke on MI was weakened in the two sample MR analysis using six SNP instruments, which excluded SNPs associated with diabetes, hypertension, atrial fibrillation, and coronary heart disease, indicating that the causal effect of all ischemic stroke on MI may be mediated by these factors.

Regarding the effect of LAS on MI, causality persisted after using the three SNP instruments, which excluded a SNP (rs2107595) that had been associated with self-reported hypertension or coronary artery disease, indicating that their causal association is robust. This study provides evidence supporting a causal relationship of genetically predicted LAS on the risk of MI using the two-sample MR method, which may control unmeasured confounders and reverse causation. ${ }^{13}$ This is a significant finding that may help to clarify the internal association between LAS and MI. As is well-known, LAS subtype and MI share many common atherosclerotic factors, ${ }^{31}$ which may indicate that there is a correlation between them. Brain injury can change the autonomic nervous and neurohormonal pathways involved in the control of heart function, 5,32 so stroke patients are prone to serious adverse cardiac events that further affect cardiac function and promote myocardial injury. From a mechanistic and pathophysiological perspective, the main reasons for the increased risk of MI after stroke are as follows: changes in the structure or function of the central autonomic neural network ${ }^{33}$ (especially the activation of the renin-angiotensin-aldosterone system); microvascular dysfunction $;{ }^{34}$ systemic proinflammatory response, ${ }^{35}$ and coronary artery demand ischemia. ${ }^{6}$ Given the high clinical burden of MI after ischemic stroke, ${ }^{36}$ high-quality monitoring of cardiovascular adverse events should be maintained for stroke patients. This research emphasizes the importance of the diagnosis and treatment of LAS and its causes, not only to prevent the recurrence of stroke but also the occurrence of myocardial infarction.

Prospective observational studies have demonstrated that MI is associated with stroke. ${ }^{3}$ Stroke is a serious complication of MI, and there is a high risk of ischemic stroke within one year after MI. ${ }^{8}$ In addition, ischemic stroke after MI is related to high mortality and morbidity, resulting in a heavy clinical burden. ${ }^{37}$ Left ventricle akinesia and/or dyskinesia after MI possibly increase the risk of mural thrombosis and cause stroke or peripheral thromboembolism. ${ }^{9}$ However, in this study, MI showed no causal effect on stroke and its subtypes using the two-sample MR method. This result suggests that the relationship between MI and stroke risk detected in observational cohort studies may be confounded by common risk factors, rather than indicates causality. As is well- known, MI and stroke have numerous common vascular risk factors, such as age, smoking, obesity, lipid disorders, diabetes mellitus, hypertension, heart failure, and atrial fibrillation. ${ }^{38}$ As our results demonstrate, after excluding SNPs related to these risk factors, the causal effect of MI on stroke is not significant. Therefore, it is critical to manage risk factors, in order to prevent the occurrence of stroke after MI. However, the summary level of the GWAS dataset can hardly eliminate the effect of other confounding factors and avoid the subthreshold effect of the genetic variants. Therefore, the causal effect of MI on stroke and its subtypes needs to be further investigated.

This study has some limitations. First, stroke and MI share many common risk factors; although we screened each SNP, there are still some potential factors that cannot be adjusted. Second, the potential horizontal pleiotropy cannot be entirely excluded, which may cause biased causal effect estimates, although pleiotropic effects were not found in the test of this study. Third, the subjects of this MR study were of European ancestry, and it cannot be completely ruled out that population stratification may have some influence on the results. Thus, this conclusion should not be generalized to other ethnic groups worldwide. Finally, there are only a few SNPs associated with LAS at a genome-wide significance level, and they cannot reliably detect the abovementioned pleiotropy with the alternative MR methods. Thus, functional SNPs in the upto-date GWASs should be used for further MR analysis to confirm the conclusion.

\section{Conclusion}

This MR analysis provides evidence to support the causal effect of LAS subtype on MI, and some factors act as confiding factors whereas others may act as mediators.

\section{Data Sharing Statement}

In this study, all data were derived from publicly available Open GWAS project database (https://gwas.mrcieu.ac.uk/), and all data can be obtained for free.

\section{Ethics Statement}

All data in this study were obtained from Open GWAS project database (https://gwas.mrcieu.ac.uk/), which has been published previously. This article does not include any studies with human participants or animals performed by any of the authors, therefore ethical approval was not needed. 


\section{Acknowledgments}

We are grateful to the investigators of MEGASTROKE and CARDIoGRAMplusC4D consortia for making their results publicly available, and thank all participants for their contribution to this study.

\section{Funding}

This research was supported by the National Key Research and Development Program, Major Chronic NonCommunicable Disease Prevention and Control Research Key Special Project (2017YFC1308202), and Henan Provincial Medical Science and Technology Research Plan (SBGJ2018031).

\section{Disclosure}

The authors report no conflicts of interest relevant to this work.

\section{References}

1. Feigin VL, Nguyen G, Cercy $\mathrm{K}$, et al. Global, regional, and country-specific lifetime risks of stroke, 1990 and 2016. $N$ Engl J Med. 2018;379(25):2429-2437.

2. Reed GW, Rossi JE, Cannon CP. Acute myocardial infarction. Lancet. 2017;389(10065):197-210. doi:10.1016/S0140-6736(16) 30677-8

3. Witt BJ, Ballman KV, Brown RD, Meverden RA, Jacobsen SJ, Roger VL. The incidence of stroke after myocardial infarction: a meta-analysis. Am J Med. 2006;119(4):354, e351-e359. doi:10.1016/j.amjmed.2005.10.058

4. Dhamoon MS, Sciacca RR, Rundek T, Sacco RL, Elkind MS. Recurrent stroke and cardiac risks after first ischemic stroke: the Northern Manhattan Study. Neurology. 2006;66(5):641-646. doi:10.1212/01.wnl.0000201253.93811.f6

5. Chen Z, Venkat P, Seyfried D, Chopp M, Yan T, Chen J. Brain-heart interaction: cardiac complications after stroke. Circ Res. 2017;121 (4):451-468. doi:10.1161/CIRCRESAHA.117.311170

6. Scheitz JF, Nolte CH, Doehner W, Hachinski V, Endres M. Stroke-heart syndrome: clinical presentation and underlying mechanisms. Lancet Neurol. 2018;17(12):1109-1120. doi:10.1016/S1474-4422(18)30336-3

7. Ferreira JP, Girerd N, Gregson J, et al. Stroke risk in patients with reduced ejection fraction after myocardial infarction without atrial fibrillation. J Am Coll Cardiol. 2018;71(7):727-735. doi:10.1016/j. jacc.2017.12.011

8. Ulvenstam A, Kajermo U, Modica A, Jernberg T, Söderström L, Mooe T. Incidence, trends, and predictors of ischemic stroke 1 year after an acute myocardial infarction. Stroke. 2014;45(11):3263-3268. doi:10.1161/STROKEAHA.114.005770

9. Yeung W, Sia CH, Pollard T, et al. Predicting mortality, thrombus recurrence and persistence in patients with post-acute myocardial infarction left ventricular thrombus. $J$ Thromb Thrombolysis. 2021;52(2):654-661. doi:10.1007/s11239-020-02368-1

10. Vandenbroucke JP. When are observational studies as credible as randomised trials? Lancet. 2004;363(9422):1728-1731. doi:10.1016/ S0140-6736(04)16261-2

11. Gupta V, Walia GK, Sachdeva MP. 'Mendelian randomization': an approach for exploring causal relations in epidemiology. Public Health. 2017;145:113-119. doi:10.1016/j.puhe.2016.12.033
12. Smith GD, Ebrahim S. 'Mendelian randomization': can genetic epidemiology contribute to understanding environmental determinants of disease? Int J Epidemiol. 2003;32(1):1-22. doi:10.1093/ije/dyg070

13. Lawlor DA, Harbord RM, Sterne JA, Timpson N, Davey smith G. Mendelian randomization: using genes as instruments for making causal inferences in epidemiology. Stat Med. 2008;27 (8):1133-1163. doi:10.1002/sim.3034

14. Davies NM, Holmes MV, Davey Smith G. Reading Mendelian randomisation studies: a guide, glossary, and checklist for clinicians. BMJ. 2018;362:k601. doi:10.1136/bmj.k601

15. Emdin CA, Khera AV, Kathiresan S. Mendelian Randomization. JAMA. 2017;318(19):1925-1926. doi:10.1001/jama.2017.17219

16. Smith GD, Davies NM, Dimou N, Egger M, Yarmolinsky J STROBE-MR: guidelines for strengthening the reporting of Mendelian randomization studies. 2019.

17. Powers WJ, Rabinstein AA, Ackerson T, et al. 2018 Guidelines for the early management of patients with acute ischemic stroke: a guideline for healthcare professionals from the American heart association/American stroke association. Stroke. 2018;49(3):e46e110. doi:10.1161/STR.0000000000000158

18. Levine GN, Bates ER, Bittl JA, et al. 2016 ACC/AHA guideline focused update on duration of dual antiplatelet therapy in patients with coronary artery disease: a report of the American college of cardiology/American heart association task force on clinical practice guidelines: an update of the 2011 ACCF/AHA/SCAI Guideline for Percutaneous Coronary Intervention, 2011 ACCF/AHA guideline for coronary artery bypass graft surgery, 2012 ACC/AHA/ACP/AATS/ PCNA/SCAI/STS guideline for the diagnosis and management of patients with stable ischemic heart disease, $2013 \mathrm{ACCF} / \mathrm{AHA}$ guideline for the management of ST-elevation myocardial infarction, 2014 AHA/ACC guideline for the management of patients with non-STelevation acute coronary syndromes, and 2014 ACC/AHA guideline on perioperative cardiovascular evaluation and management of patients undergoing noncardiac surgery. Circulation. 2016;134(10): e123-e155. doi:10.1161/CIR.0000000000000404

19. Malik R, Chauhan G, Traylor M, et al. Multiancestry genome-wide association study of 520,000 subjects identifies 32 loci associated with stroke and stroke subtypes. Nat Genet. 2018;50(4):524-537. doi:10.1038/s41588-018-0058-3

20. Nikpay M, Goel A, Won HH, et al. A comprehensive 1000 genomes-based genome-wide association meta-analysis of coronary artery disease. Nat Genet. 2015;47(10):1121-1130.

21. Machiela MJ, Chanock SJ. LDlink: a web-based application for exploring population-specific haplotype structure and linking correlated alleles of possible functional variants. Bioinformatics. 2015;31 (21):3555-3557. doi:10.1093/bioinformatics/btv402

22. Staley JR, Blackshaw J, Kamat MA, et al. PhenoScanner: a database of human genotype-phenotype associations. Bioinformatics. 2016;32 (20):3207-3209. doi:10.1093/bioinformatics/btw373

23. Kamat MA, Blackshaw JA, Young R, et al. PhenoScanner V2: an expanded tool for searching human genotype-phenotype associations. Bioinformatics. 2019;35(22):4851-4853. doi:10.1093/bioinformatics/ btz469

24. Burgess S, Thompson SG. Avoiding bias from weak instruments in Mendelian randomization studies. Int $J$ Epidemiol. 2011;40 (3):755-764. doi:10.1093/ije/dyr036

25. Hemani G, Zheng J, Elsworth B, et al. The MR-Base platform supports systematic causal inference across the human phenome. eLife. 2018:7:e34408.

26. Bowden J, Davey smith G, Burgess S. Mendelian randomization with invalid instruments: effect estimation and bias detection through Egger regression. Int J Epidemiol. 2015;44(2):512-525. doi:10.1093/ije/dyv080

27. Bowden J, Davey Smith G, Haycock PC, Burgess S. Consistent estimation in Mendelian randomization with some invalid instruments using a weighted median estimator. Genet Epidemiol. 2016;40(4):304-314. doi:10.1002/gepi.21965 
28. Touzé E, Varenne O, Chatellier G, Peyrard S, Rothwell PM, Mas JL. Risk of myocardial infarction and vascular death after transient ischemic attack and ischemic stroke: a systematic review and meta-analysis. Stroke. 2005;36(12):2748-2755. doi:10.1161/01. STR.0000190118.02275.33

29. Kang K, Park TH, Kim N, et al. Recurrent stroke, myocardial infarction, and major vascular events during the first year after acute ischemic stroke: the multicenter prospective observational study about recurrence and its determinants after acute ischemic stroke I. J Stroke Cerebrovasc Dis. 2016;25(3):656-664. doi:10.1016/j.jstrokecerebrovasdis.2015.11.036

30. Lee KJ, Kim SE, Kim JY, et al. Five-year risk of acute myocardial infarction after acute ischemic stroke in Korea. $J$ Am Heart Assoc. 2021;10(1):e018807. doi:10.1161/JAHA.120.018807

31. Frostegård J. Immunity, atherosclerosis and cardiovascular disease. BMC Med. 2013;11:117. doi:10.1186/1741-7015-11-117

32. Battaglini D, Robba C, Lopes da Silva A, et al. Brain-heart interaction after acute ischemic stroke. Crit Care. 2020;24(1):163. doi:10.1186/s13054-020-02885-8

33. Palma JA, Benarroch EE. Neural control of the heart: recent concepts and clinical correlations. Neurology. 2014;83(3):261-271. doi:10.1212/WNL.0000000000000605
34. Pelliccia F, Kaski JC, Crea F, Camici PG. Pathophysiology of Takotsubo syndrome. Circulation. 2017;135(24):2426-2441. doi:10.1161/CIRCULATIONAHA.116.027121

35. Anrather J, Iadecola C. Inflammation and stroke: an overview. Neurotherapeutics. 2016;13(4):661-670. doi:10.1007/s13311-0160483-x

36. Alqahtani F, Aljohani S, Tarabishy A, Busu T, Adcock A, Alkhouli M. Incidence and outcomes of myocardial infarction in patients admitted with acute ischemic stroke. Stroke. 2017;48 (11):2931-2938. doi:10.1161/STROKEAHA.117.018408

37. Brammås A, Jakobsson S, Ulvenstam A, Mooe T. Mortality after ischemic stroke in patients with acute myocardial infarction: predictors and trends over time in Sweden. Stroke. 2013;44(11):3050-3055. doi:10.1161/STROKEAHA.113.001434

38. Mozaffarian D, Benjamin EJ, Go AS, et al. Heart disease and stroke statistics-2016 update: a report from the American heart association. Circulation. 2016;133(4):e38-360. doi:10.1161/CIR.000000000 0000350
International Journal of General Medicine

\section{Publish your work in this journal}

The International Journal of General Medicine is an international, peer-reviewed open-access journal that focuses on general and internal medicine, pathogenesis, epidemiology, diagnosis, monitoring and treatment protocols. The journal is characterized by the rapid reporting of reviews, original research and clinical studies

\section{Dovepress}

across all disease areas. The manuscript management system is completely online and includes a very quick and fair peer-review system, which is all easy to use. Visit http://www.dovepress.com/ testimonials.php to read real quotes from published authors. 